\title{
Gender Differences in Depression in the General Population of Indonesia: Confounding Effects
}

\author{
Andi Agus Mumang $\mathbb{D}^{1},{ }^{1}$ Saidah Syamsuddin, ${ }^{2}$ Ida Leida Maria, ${ }^{3}$ and Irawan Yusuf ${ }^{4}$ \\ ${ }^{1}$ Medical Faculty Postgraduate (Doctoral) Program, Hasanuddin University, Makassar, South Sulawesi, Indonesia \\ ${ }^{2}$ Department of Psychiatry, Medical Faculty, Hasanuddin University, Makassar, South Sulawesi, Indonesia \\ ${ }^{3}$ Department of Epidemiology, Public Health Faculty, Hasanuddin University, Makassar, South Sulawesi, Indonesia \\ ${ }^{4}$ Department of Physiology, Medical Faculty, Hasanuddin University, Makassar, South Sulawesi, Indonesia
}

Correspondence should be addressed to Andi Agus Mumang; andiagusmumang93@gmail.com

Received 9 April 2021; Accepted 19 June 2021; Published 25 June 2021

Academic Editor: Giovanni Galeoto

Copyright (c) 2021 Andi Agus Mumang et al. This is an open access article distributed under the Creative Commons Attribution License, which permits unrestricted use, distribution, and reproduction in any medium, provided the original work is properly cited.

\begin{abstract}
Background. Research findings on gender differences in depression are inconsistent. This study investigated gender and depression in the Indonesian population and considered possible confounding effects. Methods. This was a cross-sectional study. Participants completed the following self-report measures: demographic characteristic questions, the Cultural Orientation Scale, and the Center for Epidemiological Studies Depression Scale. Gender differences in depression were examined using a generalized linear model. Results. After withdrawals, 265 men and 243 women remained. Women and men did not differ in overall scores and four-factor depression symptoms even after adjusting for cultural orientation and demographic confounding factors, except for the depression symptoms "crying," "cannot get going," and "people were unfriendly." Gender differences in depression became significant after adjusting for stereotypical symptom variance. Men reported being lonelier than women. Conclusions. Possible confounding effects on the association between gender and depression are methodological issues, cultural orientation transition, and stereotypical symptoms. Low depression scores found for gender may reflect dimension-counterpart coping strategies.
\end{abstract}

\section{Introduction}

Findings on gender differences in depression are inconsistent. Some studies indicate that women have a higher prevalence of depression than men [1-5], whereas others report no gender differences in depression [6-8]. There is also evidence that more men than women report depression in some situations $[9,10]$.

There are several explanations offered for gender differences in depression, particularly for why women report more depression [4, 11-13]. However, these explanations may not take into account the above-mentioned conflicting findings (see also reviews of Parker and Brotchie [14] and Piccinelli and Wilkinson [15]), which indicate inconsistencies in gender differences in depression.

Gender differences may arise from constraints and flexibility in gender socialization, which may correlate with the psychodynamics of depression $[9,16]$. Socialization con- straints are shaped by gender-typed attributes (e.g., prototype or stereotype); flexibility refers to contextual influences (e.g., sociocultural aspects) [16]. These factors have implications for confounding effects on gender differences in depression. Adherence to gender-congruent stereotypes $[17,18]$ may lead to differences in prejudice [19] and cultural orientation (i.e., arising from the interaction between horizontal-vertical and individualism-collectivism dimensions) [20,21]. The horizontal-vertical dimension reflects equality and hierarchy [22]. Therefore, gender differences in depression may reflect gender socialization, which may shape responsiveness to sociocultural situations (e.g., cultural transition and acculturation) $[20,21,23-25]$.

More studies have focused on depression in women than depression in men [25]; consequently, male depression has been neglected [18]. Additionally, the overreporting of depression by women and underreporting by men is an artifact of gender-difference research [26, 27]. Moreover, 
TABLE 1: Demographic characteristics and cultural orientation.

\begin{tabular}{|c|c|c|c|}
\hline Variables & Women $=243$ & Men $=265$ & $p$ value \\
\hline \multicolumn{4}{|l|}{ Demographic characteristics } \\
\hline Age & $36.65 \pm 11.83$ & $32.63 \pm 11.23$ & $<0.001$ \\
\hline \multicolumn{4}{|l|}{ Ethnicity } \\
\hline Bugis/Makassar/mixed & 86.4 & 85.3 & 0.714 \\
\hline Other & 13.6 & 14.7 & \\
\hline \multicolumn{4}{|l|}{ Education level } \\
\hline Low & 16.9 & 4.9 & $<0.001$ \\
\hline Medium & 28.8 & 22.6 & \\
\hline High & 54.3 & 72.5 & \\
\hline \multicolumn{4}{|l|}{ Occupation } \\
\hline Civil servant & 7.8 & 11.7 & $<0.001$ \\
\hline Self-employed & 31.7 & 47.2 & \\
\hline Other ${ }^{\mathrm{a}}$ & 60.5 & 41.1 & \\
\hline \multicolumn{4}{|l|}{ Household income level } \\
\hline Low & 32.5 & 25.7 & $<0.001$ \\
\hline Medium & 44.4 & 59.2 & \\
\hline High & 5.3 & 10.2 & \\
\hline Unknown & 17.7 & 4.9 & \\
\hline \multicolumn{4}{|l|}{ Cultural orientation } \\
\hline Horizontal individualism (HI) $(\alpha=0.849)$ & $26.33 \pm 7.96$ & $24.92 \pm 7.08$ & 0.003 \\
\hline Vertical individualism (VI) $(\alpha=0.761)$ & $25.20 \pm 7.17$ & $24.82 \pm 6.69$ & 0.277 \\
\hline Horizontal collectivism $(\mathrm{HC})(\alpha=0.738)$ & $25.71 \pm 6.80$ & $26.00 \pm 5.49$ & 0.650 \\
\hline Vertical collectivism (VC) $(\alpha=0.863)$ & $30.35 \pm 6.57$ & $28.69 \pm 5.90$ & $<0.001$ \\
\hline
\end{tabular}

Data are means \pm standard deviations and percentages and were analyzed using chi-square and Mann-Whitney $U$ tests $(\alpha=0.05)$. The reliability was analyzed using Cronbach's alpha $(\alpha)$. 'Occupations with low frequencies were categorized as "other".

perceived gender differences in depression may be a result of gender stereotypes $[28,29]$ or methodological issues $[9,18$, 27]. These factors may explain confounding effects on gender differences, particularly the higher prevalence of depression in women.

Previous epidemiological studies have analyzed gender and depression in representative Indonesian populations [7, 8 ]. However, they did not focus on gender and do not provide a clear explanation of gender differences in depression. Therefore, this study investigated gender differences in depression in Indonesia, particularly the possible role of confounding effects.

\section{Methods}

2.1. Participants. From June to November 2019, a crosssectional study was conducted among the general population in Indonesia, which followed the STROBE Statement (https://www.strobe-statement.org/index.php?id=availablechecklists) for observational studies. For convenience, the sample comprised individuals living up to $10 \mathrm{~km}$ from Hasanuddin University, Makassar, South Sulawesi. This study enrolled 300 male and 300 female volunteers. Participants were given self-report questionnaires after providing written informed consent (response rates for men and women: $88.3 \%$ and $81 \%$, respectively). Participants who resigned from par- ticipation during the sampling process or did not return their questionnaires were withdrawn from the study. The ethics commission of the Faculty of Medicine at Hasanuddin University approved all procedures (approval number: 616/UN4.6.4.5.31/PP36/2019).

2.2. Assessments. The questionnaires comprised items on demographic characteristics, the Cultural Orientation Scale (COS) [22], and the Center for Epidemiological Studies Depression Scale (CES-D) [30]. Both the COS and the CES-D are appropriate measures of dimensions (horizontal-vertical) of individualism-collectivism cultural orientation and perceived depression symptoms, respectively [8, 31-33]. The COS dimensions are horizontal individualism (HI; my personal identity, independent of others, is very important to me; $\alpha=0.849$ ), vertical individualism (VI; competition is the law of nature; $\alpha=0.761)$, horizontal collectivism (HC; I feel good when I cooperate with others; $\alpha=0.738$ ), and vertical collectivism (VC; parents and children must stay together as much as possible; $\alpha=0.863$ ). The 20-item CES-D $(\alpha=0.812)$ has four factors [30, 34]: depressed affect (e.g., I felt depressed), positive affect (e.g., I felt that I was just as good as other people), somatic and retarded activity (e.g., my sleep was restless), and interpersonal (e.g., people were unfriendly). The reliability of the scales was analyzed using Cronbach's alpha. 
TABLE 2: Mean gender differences in overall and four-factor depression symptoms.

\begin{tabular}{|c|c|c|c|}
\hline Depression symptoms (CES-D) & Women $=243$ & Men $=265$ & $p$ value \\
\hline Overall $(0-60)^{\dagger}$ & $14.71 \pm 8.00$ & $13.98 \pm 7.82$ & 0.237 \\
\hline Depressed affect (0-21) & $3.76 \pm 3.79$ & $3.42 \pm 2.82$ & 0.196 \\
\hline Blues $(03)^{\dagger \dagger}$ & $0.55 \pm 0.84$ & $0.61 \pm 0.87$ & 0.407 \\
\hline Depressed (06) & $0.54 \pm 0.82$ & $0.58 \pm 0.82$ & 0.451 \\
\hline Failure (09) & $0.36 \pm 0.72$ & $0.34 \pm 0.76$ & 0.609 \\
\hline Fearful (10) & $0.53 \pm 0.75$ & $0.45 \pm 0.76$ & 0.100 \\
\hline Lonely (14) & $0.55 \pm 0.84$ & $0.61 \pm 0.84$ & 0.296 \\
\hline Crying (17) & $0.54 \pm 0.85$ & $0.33 \pm 0.66$ & 0.011 \\
\hline Sad (18) & $0.69 \pm 0.88$ & $0.50 \pm 0.76$ & 0.015 \\
\hline Positive affect ${ }^{\mathrm{a}}(0-12)$ & $3.28 \pm 3.02$ & $3.05 \pm 2.56$ & 0.805 \\
\hline Good (04) & $0.87 \pm 1.06$ & $0.79 \pm 0.99$ & 0.580 \\
\hline Hopeful (08) & $0.81 \pm 1.01$ & $0.86 \pm 0.94$ & 0.233 \\
\hline Happy (12) & $0.89 \pm 0.90$ & $0.81 \pm 0.84$ & 0.345 \\
\hline Enjoy (16) & $0.71 \pm 1.01$ & $0.59 \pm 0.83$ & 0.544 \\
\hline Somatic and retarded activity $(0-21)$ & $6.37 \pm 3.47$ & $6.40 \pm 2.98$ & 0.761 \\
\hline Bothered (01) & $0.54 \pm 0.81$ & $0.49 \pm 0.70$ & 0.974 \\
\hline Appetite (02) & $0.66 \pm 0.83$ & $0.66 \pm 0.79$ & 0.867 \\
\hline Mind (05) & $0.97 \pm 0.92$ & $1.04 \pm 0.89$ & 0.245 \\
\hline Effort (07) & $2.23 \pm 0.98$ & $2.28 \pm 0.94$ & 0.680 \\
\hline Sleep (11) & $0.71 \pm 0.91$ & $0.68 \pm 0.89$ & 0.852 \\
\hline Talk (13) & $0.79 \pm 0.86$ & $0.87 \pm 0.88$ & 0.330 \\
\hline Get going (20) & $0.48 \pm 0.50$ & $0.37 \pm 0.48$ & 0.018 \\
\hline Interpersonal (0-6) & $1.30 \pm 1.55$ & $1.10 \pm 1.29$ & 0.370 \\
\hline Unfriendly (15) & $0.76 \pm 1.02$ & $0.54 \pm 0.85$ & 0.030 \\
\hline Dislike (19) & $0.55 \pm 0.83$ & $0.57 \pm 0.78$ & 0.484 \\
\hline
\end{tabular}

Mann-Whitney $U$ test with $\alpha=0.05$. Cronbach's alpha $(\alpha)$ for the CES-D was 0.812. Abbreviation: CES-D: Center for Epidemiological Studies Depression

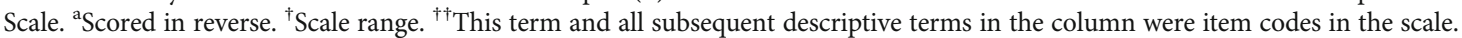

2.3. Analysis Procedures. Proportions and mean differences were analyzed using the nonparametric chi-square $\left(\chi^{2}\right)$ and Mann-Whitney $U$ tests. A generalized linear model was performed for multivariate analysis of gender differences in depression, adjusting for cultural orientation. Furthermore, the possible effects of stereotypical symptoms were identified by adjusting their variance by either controlling or not controlling for stereotypical symptom variances in the analysis. When not controlling for stereotypical symptom variances, other symptom variances were controlled for. All analyses controlled for the potential confounding effects of demographic characteristics, and "women" was the reference category. Statistical tests were two-sided with a $95 \%$ confidence interval ( $\mathrm{CI} ; \alpha=0.05)$. IBM SPSS for Windows, Version 26.0 was used for analysis (IBM Corp., Armonk, NY, USA).

\section{Results}

After withdrawals ( 35 men, 57 women), 265 men and 243 women remained. There were significant gender differences in most demographic characteristics (all $p<0.001$ ) except ethnicity $(p=0.714)$; in cultural orientation, HI $(p=0.003)$ and VC $(p<0.001)$ evidenced significant gender differences (Table 1$)$.

Table 2 shows no gender difference in overall depression scores $(p=0.273)$ and in scores on the four depression factors (depressed affect, $p=0.196$; positive affect, $p=0.805$; somatic and retarded activity, $p=0.761$; interpersonal, $p=$ $0.370)$. The only gender differences were found in the symptoms, "crying" $(p=0.011)$, "sad" $(p=0.015)$, "cannot get going" ( $p=0.018)$, and "people were unfriendly" ( $p=0.030)$.

Table 3 shows the multivariate analysis of gender differences in depression. After controlling for potential demographic confounding, no gender differences were found, after adjusting for cultural orientation in the overall and four-factor depression symptoms. However, the symptoms "crying," "cannot get going," and "people were unfriendly" remained significant (adjusted odds ratio $($ ORadj $)=0.597$; $95 \% \mathrm{CI}=0.404-0.828, \quad p<0.01, \quad$ ORadj $=0.411 ; 95 \% \mathrm{CI}=$ $0.230-0.733, \quad p<0.05, \quad$ ORadj $=0.748 ; \quad 95 \% \mathrm{CI}=0.581-$ $0.963, p<0.05$, respectively). Men had a higher OR than women for overall scores $(\mathrm{ORadj}=1.077 ; 95 \% \mathrm{CI}=1.031$ - 
TABLE 3: Multivariate analysis of gender differences in overall and four-factor depression symptoms.

\begin{tabular}{|c|c|c|}
\hline Variables & $\mathrm{B}(\mathrm{SE})$ & OR $(95 \% \mathrm{CI})$ \\
\hline \multicolumn{3}{|l|}{ Overall symptoms } \\
\hline Adj. for cultural orientation ${ }^{a}$ & $-0.002(0.013)$ & $0.998(0.973-1.024)$ \\
\hline Adj. for cultural orientation +3 -item stereotypes ${ }^{b}$ & $0.075(0.023)$ & $1.077(1.031-1.126)^{* *}$ \\
\hline Adj. for cultural orientation +17 items $^{c}$ & $-0.442(0.101)$ & $0.643(0.527-0.783)^{* * *}$ \\
\hline \multicolumn{3}{|c|}{${ }^{\mathrm{a}} \mathrm{AIC}=676.147, \mathrm{BIC}=722.683 ;{ }^{\mathrm{b}} \mathrm{AIC}=662.849, \mathrm{BIC}=722.076 ;{ }^{\mathrm{c}} \mathrm{AIC}=663.671, \mathrm{BIC}=782.125$} \\
\hline \multicolumn{3}{|l|}{ Four factors } \\
\hline \multicolumn{3}{|l|}{ Adj. for cultural orientation } \\
\hline Depressed affect & $0.003(0.047)$ & $1.004(0.916-1.099)$ \\
\hline Positive affect & $-0.008(0.038)$ & $0.992(0.921-1.068)$ \\
\hline Somatic and retarded activity & $0.022(0.055)$ & $1.021(0.917-1.139)$ \\
\hline Interpersonal & $-0.095(0.081)$ & $0.910(0.777-1.065)$ \\
\hline \multicolumn{3}{|c|}{$\mathrm{AIC}=680.490, \mathrm{BIC}=739.717$} \\
\hline \multicolumn{3}{|l|}{ Adj. for cultural orientation +3 -item stereotypes } \\
\hline Depressed affect & $0.171(0.066)$ & $1.186(1.043-1.350)^{* *}$ \\
\hline Positive affect & $-0.004(0.039)$ & $0.996(0.922-1.076)$ \\
\hline Somatic and retarded activity & $0.118(0.063)$ & $1.125(0.994-1.274)$ \\
\hline Interpersonal & $0.105(0.143)$ & $1.110(0.839-1.470)$ \\
\hline \multicolumn{3}{|c|}{$\mathrm{AIC}=665.942, \mathrm{BIC}=737.861$} \\
\hline \multicolumn{3}{|l|}{ Adj. for cultural orientation +17 items } \\
\hline Depressed affect & $-0.607(0.176)$ & $0.545(0.386-0.769)^{* *}$ \\
\hline Positive affect & $-0.207(0.154)$ & $0.813(0.601-1.100)$ \\
\hline Somatic and retarded activity & $-0.639(0.282)$ & $0.528(0.304-0.918)^{*}$ \\
\hline Interpersonal & $-0.294(0.129)$ & $0.745(0.579-0.960)^{*}$ \\
\hline \multicolumn{3}{|c|}{$\mathrm{AIC}=663.987, \mathrm{BIC}=790.902$} \\
\hline \multicolumn{3}{|l|}{ Items on the four factors } \\
\hline \multicolumn{3}{|l|}{ Depressed affect } \\
\hline Blues & $0.200(0.148)$ & $1.221(0.913-1.634)$ \\
\hline Depressed & $0.321(0.167)$ & $1.379(0.993-1.915)$ \\
\hline Failure & $0.189(0.189)$ & $1.208(0.833-1.753)$ \\
\hline Fearful & $-0.251(0.181)$ & $0.788(0.546-1.110)$ \\
\hline Lonely & $0.381(0.148)$ & $1.464(1.083-1.978)^{*}$ \\
\hline Sad & $-0.258(0.180)$ & $0.773(0.534-1.118)$ \\
\hline \multicolumn{3}{|l|}{ Positive affect } \\
\hline Good & $-0.017(0.122)$ & $0.983(0.774-1.248)$ \\
\hline Hopeful & $0.200(0.126)$ & $1.221(0.955-1.562)$ \\
\hline Happy & $-0.096(0.153)$ & $0.908(0.674-1.225)$ \\
\hline Enjoy & $-0.191(0.154)$ & $0.826(0.611-1.116)$ \\
\hline \multicolumn{3}{|l|}{ Somatic and retarded activity } \\
\hline Bothered & $-0.240(0.153)$ & $0.787(0.583-1.061)$ \\
\hline Appetite & $0.204(0.148)$ & $1.226(0.916-1.640)$ \\
\hline Mind & $0.204(0.133)$ & $1.227(0.945-1.593)$ \\
\hline Effort & $0.059(0.136)$ & $1.061(0.812-1.386)$ \\
\hline Sleep & $0.293(0.153)$ & $1.340(0.994-1.808)$ \\
\hline Talk & $0.248(0.135)$ & $1.281(0.983-1.670)$ \\
\hline \multicolumn{3}{|l|}{ Interpersonal } \\
\hline Dislike & $0.224(0.158)$ & $1.251(0.918-1.248)$ \\
\hline 3-item stereotypes & & \\
\hline
\end{tabular}


TABLE 3: Continued.

\begin{tabular}{lrr}
\hline Variables & B (SE) & OR (95\% CI) \\
\hline Crying & $-0.547(0.178)$ & $0.597(0.404-0.828)^{* *}$ \\
Get going & $-0.890(0.296)$ & $0.411(0.230-0.733)^{*}$ \\
Unfriendly & $-0.290(0.128)$ & $0.748(0.581-0.963)^{*}$ \\
Cultural orientation & \\
Horizontal individualism (HI) & $-0.039(0.018)$ & $0.962(0.928-0.996)^{*}$ \\
Vertical individualism (VI) & $0.026(0.019)$ & $1.025(0.985-1.065)$ \\
Horizontal collectivism (HC) & $0.062(0.025)$ & $1.063(1.012-1.116)^{*}$ \\
Vertical collectivism (VC) & $-0.078(0.025)$ & $0.926(0.883-0.971)^{* *}$ \\
& AIC $=663.972, \mathrm{BIC}=790.887$ & \\
\hline
\end{tabular}

Reference category: women. Note: three items that seemed to indicate stereotypical symptoms were grouped as 3-item stereotypes. Adjusting for 3-item stereotypes and 17 items, respectively, means controlling/not controlling for mean stereotypical symptom variances in total depression scores. The scores on the four factors were not analyzed separately. Loneliness symptoms were not significant after excluding 3-item stereotypes from the analysis. HI, HC, and VC were significant in all adjusted models with no substantial change in OR (their respective results are shown at the bottom of the table). All analyses controlled for demographic confounding. The omnibus test was significant for all models $(p<0.001)$. Goodness of fit was measured using the AIC and BIC. Abbreviations: Adj.: adjusted; B: beta coefficient; SE: standard error; OR: odds ratio; CI: confidence interval; AIC: Akaike information criterion; BIC: Bayesian information criterion. ${ }^{* * *} p<0.001 .{ }^{* *} p<0.01 .{ }^{*} p<0.05$.

$1.126, p<0.01)$ and depressed affect (ORadj $=1.186 ; 95 \% \mathrm{CI}$ $=1.043-1.350, \quad p<0.01)$, particularly "loneliness" $($ ORadj $=1.464 ; \quad 95 \% \mathrm{CI}=1.083-1.978, \quad p<0.05), \quad$ after adjusting for variance on the three symptoms. Conversely, men had a lower OR than women for overall scores (ORadj $=0.643 ; 95 \% \mathrm{CI}=0.527-0.783, p<0.001)$, depressed affect (ORadj $=0.545 ; \quad 95 \% \mathrm{CI}=0.386-0.769, \quad p<0.01$ ), somatic and retarded activity (ORadj $=0.528 ; 95 \% \mathrm{CI}=$ $0.304-0.918, p<0.05$ ), and interpersonal (ORadj $=0.745$; $95 \% \mathrm{CI}=0.579-0.960, p<0.05)$, when only the 17 -item variance was adjusted.

\section{Discussion}

4.1. Methodological Confounding Effects. No gender differences were found for overall depression and for most of the four-factor depression symptoms. This finding contradicts a previous finding of gender differences in overall and fourfactor depression symptoms [35]. Hawkins et al. [36] partly support the finding in the present study of no gender differences except for depressed affect.

These contradictions may indicate methodological confounding factors (e.g., sample preferences) [9, 18], which may affect gender differences. More specifically, both previous studies enrolled samples more vulnerable to depression (married people and students) than the sample (general population) in the present study $[11,37,38]$. In support of this view, some general population studies show no gender differences in depression [6-8].

4.2. Cultural Confounding Effects. No significant gender differences were found in the odds of depression after adjusting for cultural orientation and demographic confounding factors, with one exception, which is discussed below. However, significant gender differences were found in cultural orientation (men were higher on HC and lower on VC and HI than women), which may indicate confounding effects on gender differences in depression. It is assumed that lower scores on collectivism dimensions related to gender may indicate a transition process to corresponding individualism dimensions (one-way transition). Men with relatively low VC may experience vertical transitions to VI, whereas women with relatively low $\mathrm{HC}$ may experience horizontal transitions to HI. Additionally, the significant gender differences for $\mathrm{HI}$ but not for VI may indicate that the transition process is easier on the horizontal than on the vertical dimension [24]. Although women scored slightly higher on VI than men (nonsignificant), the smaller vertical difference (VC-VI) in men could indicate rapid vertical transition.

The current sociocultural transition (collectivism to individualism) in Indonesian society, especially for those living in towns (e.g., Makassar city) [39-42], may support these assumptions. It may predispose gender responsiveness to manage socialization during the transition that fits with gender roles and value motivations [21]. Hence, men are more likely involved in vertical (hierarchy-mastery) and women in horizontal (egalitarian-harmony) dimensions [21, 23, 33, 43]. Additionally, men may require transition to VI to compete and accomplish more hierarchy-mastery rewards, to fulfill their (internalized) expectations, and to obtain selfauthorization [21, 33, 43]. For women, horizontal transition to individualism may be required to show that they are independent and strong, or as a behavioral adjustment to social change [21, 44-46].

The transition toward alternative cultural orientations may challenge gender socialization responsiveness in acculturation and cause depression because it clashes with the embedded culture [20, 47, 48]. Men who require selfauthorization or express legal nonconformity may be challenged by normative VC socialization, which emphasizes strict conformity to the authority pattern (e.g., living longer with parents/extended family, obedience to family hierarchy, and sacrifice for ingroup). Women who require selfindependence may be challenged by normative $\mathrm{HC}$ 
socialization, which emphasizes ingroup harmonization (e.g., benevolence and sociability) [21, 22, 49-51]. Challenges in socializing to their divergent cultural orientations may cause men and women to experience equivalent levels of depression. Therefore, inconsistencies in gender differences in depression may be found during the transition.

However, the higher $\mathrm{HC}$ in men and VC in women may indicate gender-based dimension-counterpart coping strategies to negotiate potential socialization difficulties. This may explain the low depression scores found in the present study (under the general threshold for substantial depression) $[30,32]$. Men who experience depression with vertical transition may overcome the situation by maintaining harmonization in socialization (e.g., cooperation and tolerance) $[52,53]$. Women who experience depression with horizontal transition may overcome the situation by limiting their goals to family preservation and responsibility, especially regarding children $[43,44]$.

4.3. Stereotypical Symptom Confounding Effects. Gender differences in depression were found in a few symptoms (crying, inability to get going, and feeling that people are unfriendly) even after adjustment. The reported higher depression prevalence in women may indicate typical features (feminine stereotypes) of depression symptoms in women, especially crying $[18,25,35,54]$, which seem incongruent with the prototypical male gender role $[17,18]$. Additionally, women's motives typically involve emotional relationships (other-oriented), whereas men's motives are typically more self-oriented [9, 55-57]. This may explain why women are more willing to report their interpersonal problems than men. Men are also more stereotypically proactive than women (e.g., they find it easier to "get going") [58, 59]. Thus, gender differences in depression may occur only in stereotypical symptoms, not in overall symptoms [28, 29], or may reflect adherence to congruent gender-role stereotypes (e.g., men should avoid all things feminine) $[17,18]$.

Adherence to traditional male roles may prompt men to suppress feminine stereotypical symptoms, which may explain why men in this study were significantly more depressed (particularly lonelier) than women, after adjusting for feminine stereotypical symptoms (i.e., adjusted 3-item stereotypes) $[10,18,42,60]$. An unwillingness to show weakness (e.g., crying and inability to get going) and an inclination to self-focused attention (indicated by low scores on the interpersonal symptom "feeling people were unfriendly") may predispose men to experience more self-isolation or social withdrawal when struggling with depression $[18,60]$. In contrast, women may express more feminine stereotypical symptoms to obtain support or as social signals (e.g., relationship reconciliation) [53, 60]. Newmann [54] has suggested that this is a gender-typical coping pattern that works better for women.

The odds of depression in women were higher for depressed affect, somatic and retarded activity, and interpersonal problems when feminine stereotypical symptoms were not controlled for (i.e., adjusted only for 17 items). This may indicate that gender differences are not real but artifacts [26, 27] and apparent only for stereotypical symptoms. This stereotypical-induced artifact may lead women to overreport depression and to therefore seem more depressed than men.

4.4. Limitations and Future Directions. There were some study limitations. First, it may be premature to assume the existence of a transition process based only on interpretation of a "decline score" in certain cultural dimensions, particularly given the limitations of the study design. Second, the sample is not representative of all residential conditions of the Makassar population, and problems of language comprehension in older participants may have led to biased information. However, these findings may provide preliminary information for further research (with better study designs and more advanced analysis) on possible cultural transition effects on gender differences in depression. Additionally, studies comparing different samples (e.g., general population vs. college students or married couples) would be useful to further investigate confounding effects on gender differences in depression. The possibility of stereotypical symptoms should be considered in gender-difference analysis and diagnosis because these may confound results. Finally, more representative sampling across different residential conditions of the population is needed.

\section{Conclusions}

No gender differences in depression were found, which may indicate methodological issues or the effects of cultural orientation transition. However, for some depression symptoms, there were significant differences in gender, which may indicate the effects of stereotypical symptoms. These may show possible confounding effects on the association between gender and depression. Additionally, the low depression scores for both genders may reflect dimension-counterpart coping strategies that people implement during cultural orientation transitions. Further research is required to build on these preliminary findings.

\section{Data Availability}

All data underlying the results are available as part of the article, and no additional source data are required. Additionally, the raw data used to support the findings of this study are available from the corresponding author upon reasonable request.

\section{Disclosure}

The funder had no role in the study design; in the collection, analysis, and interpretation of data; in the writing of the report; or in the decision to submit the article for publication.

\section{Conflicts of Interest}

The authors report no conflicts of interest.

\section{Acknowledgments}

We thank all participants and field assistants for their contributions. We thank Diane Williams, Ph.D., and Anita 
Harman, Ph.D., from Edanz (https://jp.edanz.com/ac) for editing a draft of this manuscript. This work was supported by the Ministry of Research and Technology/National Research and Innovation Agency of Republic Indonesia (RISTEK-BRIN) (grant/award numbers: 9/E1/KP.PTNBH/2019 and 7/AMD/E1/KP.PTNBH/2020).

\section{References}

[1] C. A. Essau, P. M. Lewinsohn, J. R. Seeley, and S. Sasagawa, "Gender differences in the developmental course of depression," Journal of Affective Disorders, vol. 127, no. 1-3, pp. 185-190, 2010.

[2] K. S. Kendler and C. O. Gardner, "Sex differences in the pathways to major depression: a study of opposite-sex twin pairs," The American Journal of Psychiatry, vol. 171, no. 4, pp. 426435, 2014.

[3] R. H. Salk, J. S. Hyde, and L. Y. Abramson, "Gender differences in depression in representative national samples: metaanalyses of diagnoses and symptoms," Psychological Bulletin, vol. 143, no. 8, pp. 783-822, 2017.

[4] C. Kuehner, "Why is depression more common among women than among men?," The Lancet Psychiatry, vol. 4, no. 2, pp. 146-158, 2017.

[5] L. A. Pratt and D. J. Brody, "Depression in the U.S. household population, 2009-2012," NCHS Data Brief, vol. 172, pp. 1-8, 2014.

[6] L. Qi, Y. Zhang, L. Wang et al., "Sex differences in psychotic and non-psychotic major depressive disorder in a Chinese Han population," Journal of Affective Disorders, vol. 268, pp. 55-60, 2020.

[7] B. A. Pramesona and S. Taneepanichskul, "Prevalence and risk factors of depression among Indonesian elderly: a nursing home-based cross-sectional study," Neurology Psychiatry and Brain Research, vol. 30, pp. 22-27, 2018.

[8] K. Peltzer and S. Pengpid, "High prevalence of depressive symptoms in a national sample of adults in Indonesia: childhood adversity, sociodemographic factors and health risk behaviour," Asian Journal of Psychiatry, vol. 33, pp. 52-59, 2018.

[9] S. Rosenfield, "Sex differences in depression: do women always have higher rates?," Journal of Health and Social Behavior, vol. 21, no. 1, pp. 33-42, 1980.

[10] J. J. Flynn, T. Hollenstein, and A. Mackey, "The effect of suppressing and not accepting emotions on depressive symptoms: is suppression different for men and women?," Personality and Individual Differences, vol. 49, no. 6, pp. 582-586, 2010.

[11] W. R. Gove, "The relationship between sex roles, marital status, and mental illness," Social Forces, vol. 51, no. 1, p. 34, 1972.

[12] J. S. Girgus and K. Yang, "Gender and depression," Current Opinion in Psychology, vol. 4, pp. 53-60, 2015.

[13] P. Bebbington, "The origins of sex differences in depressive disorder: bridging the gap," International Review of Psychiatry, vol. 8, no. 4, pp. 295-332, 1996.

[14] G. Parker and H. Brotchie, "Gender differences in depression," International Review of Psychiatry, vol. 22, no. 5, pp. 429-436, 2010.

[15] M. Piccinelli and G. Wilkinson, "Gender differences in depression: Critical review," The British Journal of Psychiatry, vol. 177, no. 6, pp. 486-492, 2000.
[16] W. Wood and A. H. Eagly, "A cross-cultural analysis of the behavior of women and men: implications for the origins of sex differences," Psychological Bulletin, vol. 128, no. 5, pp. 699-727, 2002.

[17] E. S. Mankowski and R. M. Smith, "Men's mental health and masculinities," in Encyclopedia of Mental Health, H. S. Friedman, Ed., pp. 66-74, Academic Press, 2016.

[18] L. W. Warren, "Male intolerance of depression: a review with implications for psychotherapy," Clinical Psychology Review, vol. 3, no. 2, pp. 147-156, 1983.

[19] I. K. Broverman, D. M. Broverman, F. E. Clarkson, P. S. Rosenkrantz, and S. R. Vogel, "Sex-role stereotypes and clinical judgments of mental health," Journal of Consulting and Clinical Psychology, vol. 34, no. 1, pp. 1-7, 1970.

[20] C. J. Falicov, "Culture, society and gender in depression," Journal of Family Therapy, vol. 25, no. 4, pp. 371-387, 2003.

[21] J. Zhang, H. Mandl, and E. Wang, "The effect of verticalhorizontal individualism-collectivism on acculturation and the moderating role of gender," International Journal of Intercultural Relations, vol. 35, no. 1, pp. 124-134, 2011.

[22] H. C. Triandis and M. J. Gelfand, "Converging measurement of horizontal and vertical individualism and collectivism," Journal of Personality and Social Psychology, vol. 74, no. 1, pp. 118-128, 1998.

[23] S. H. Schwartz and T. Rubel-Lifschitz, "Cross-national variation in the size of sex differences in values: effects of gender equality," Journal of Personality and Social Psychology, vol. 97, no. 1, pp. 171-185, 2009.

[24] V. Chirkov, R. M. Ryan, Y. Kim, and U. Kaplan, "Differentiating autonomy from individualism and independence: a selfdetermination theory perspective on internalization of cultural orientations and well-being," Journal of Personality and Social Psychology, vol. 84, no. 1, pp. 97-110, 2003.

[25] M. E. Addis, "Gender and depression in men," Clinical Psychology: Science and Practice, vol. 15, no. 3, pp. 153-168, 2008.

[26] D. L. Phillips and B. E. Segal, "Sexual status and psychiatric symptoms," American Sociological Review, vol. 34, no. 1, pp. 58-72, 1969.

[27] K. Wilhelm, "Sex differences in lifetime depression rates: fact or artefact?," Psychological Medicine, vol. 24, no. 1, pp. 97111, 1994.

[28] E. S. Chevron, D. M. Quinlan, and S. J. Blatt, "Sex roles and gender differences in the experience of depression," Journal of Abnormal Psychology, vol. 87, no. 6, pp. 680-683, 1978.

[29] A. H. Fischer, "Sex differences in emotionality: fact or stereotype," Feminism \& Psychology, vol. 3, no. 3, pp. 303-318, 1993.

[30] L. S. Radloff, "The CES-D scale," Applied Psychological Measurement, vol. 1, no. 3, pp. 385-401, 1977.

[31] A. K. Lalwani, S. Shavitt, and T. Johnson, "What is the relation between cultural orientation and socially desirable responding?," Journal of Personality and Social Psychology, vol. 90, no. 1, pp. 165-178, 2006.

[32] G. Vilagut, C. G. Forero, G. Barbaglia, and J. Alonso, "Screening for depression in the general population with the Center for Epidemiologic Studies Depression (CES-D): a systematic review with meta-analysis," PLoS One, vol. 11, no. 5, article e0155431, 2016.

[33] A. Germani, E. Delvecchio, J.-B. Li, and C. Mazzeschi, "The horizontal and vertical individualism and collectivism scale: early evidence on validation in an Italian sample," Journal of Child and Family Studies, vol. 29, no. 3, pp. 904-911, 2020. 
[34] T. D. Cosco, C. C. Lachance, J. M. Blodgett et al., "Latent structure of the Centre for Epidemiologic Studies Depression Scale (CES-D) in older adult populations: a systematic review," Aging and Mental Health, vol. 24, no. 5, pp. 700-704, 2020.

[35] C. E. Ross and J. Mirowsky, "Components of depressed mood in married men and women the center for epidemiologic studies' depression scale," American Journal of Epidemiology, vol. 119, no. 6, pp. 997-1004, 1984.

[36] W. E. Hawkins, R. J. McDermott, L. Sheilds, and S. M. Harvey, "Sex differences in the "depressed affect" factor among selected university students," Psychological Reports, vol. 64, 3_suppl, pp. 1245-1246, 1989.

[37] P. Nerdrum, T. Rustøen, and M. H. Rønnestad, "Student psychological distress: a psychometric study of 1750 Norwegian 1st-year undergraduate students," Scandinavian Journal of Educational Research, vol. 50, no. 1, pp. 95-109, 2006.

[38] L. Radloff, "Sex differences in depression," Sex Roles, vol. 1, no. 3, pp. 249-265, 1975.

[39] C. Geertz, "Culture and social change: the Indonesian case," Man, vol. 19, no. 4, pp. 511-532, 1984.

[40] W. L. H. Mangundjaya, "Is there cultural change in the national cultures of Indonesia?," The 2010 Congress of the International Association for Cross-Cultural Psychology, 2013.

[41] A. A. Mumang, K. Liaury, S. Syamsuddin et al., "Socio-economic-demographic determinants of depression in Indonesia: a hospital-based study," PLoS One, vol. 15, no. 12, article e0244108, 2020.

[42] Y. Lu, "Mental health and risk behaviours of rural urban migrants: longitudinal evidence from Indonesia," Population Studies, vol. 64, no. 2, pp. 147-163, 2010.

[43] M. Kalkan and H. Odac1, "Endorsement of traditional male role norms and marital adjustment among Turkish men," Men and Masculinities, vol. 20, no. 2, pp. 135-153, 2017.

[44] B. W. Roberts and R. Helson, "Changes in culture, changes in personality: the influence of individualism in a longitudinal study of women," Journal of Personality and Social Psychology, vol. 72, no. 3, pp. 641-651, 1997.

[45] A. M. Pirttilä-Backman, B. R. Kassea, and T. Ikonen, "Cameroonian forms of collectivism and individualism," Journal of Cross-Cultural Psychology, vol. 35, no. 4, pp. 481-498, 2004.

[46] T. Beauboeuf-Lafontant, "You have to show strength," Gender and Society, vol. 21, no. 1, pp. 28-51, 2007.

[47] E. I. Lorenzo-Blanco, J. B. Unger, L. Baezconde-Garbanati, A. Ritt-Olson, and D. Soto, "Acculturation, enculturation, and symptoms of depression in Hispanic youth: the roles of gender, Hispanic cultural values, and family functioning," Journal of Youth and Adolescence, vol. 41, no. 10, pp. 13501365, 2012.

[48] C. L. Caldwell-Harris and A. Ayçiçegi, "When personality and culture clash: the psychological distress of allocentrics in an individualist culture and idiocentrics in a collectivist culture," Transcultural Psychiatry, vol. 43, no. 3, pp. 331-361, 2006.

[49] D. C. Thomas and A. A. Pekerti, "Effect of culture on situational determinants of exchange behavior in organizations," Journal of Cross-Cultural Psychology, vol. 34, no. 3, pp. 269281, 2003.

[50] Y. E. Riany, P. Meredith, and M. Cuskelly, "Understanding the influence of traditional cultural values on Indonesian parenting," Marriage and Family Review, vol. 53, no. 3, pp. 207226, 2017.
[51] D. R. Sawitri and P. A. Creed, "Collectivism and perceived congruence with parents as antecedents to career aspirations," Journal of Career Development, vol. 44, no. 6, pp. 530-543, 2017.

[52] J. F. Benenson, H. Markovits, C. Fitzgerald et al., "Males' greater tolerance of same-sex peers," Psychological Science, vol. 20, no. 2, pp. 184-190, 2009.

[53] L. Oppenheimer, "Perception of individualism and collectivism in Dutch society: a developmental approach," International Journal of Behavioral Development, vol. 28, no. 4, pp. 336-346, 2004.

[54] J. P. Newmann, "Gender, life strains, and depression," Journal of Health and Social Behavior, vol. 27, no. 2, pp. 161-178, 1986.

[55] E. G. T. Green, J. C. Deschamps, and D. Páez, "Variation of individualism and collectivism within and between 20 countries," Journal of Cross-Cultural Psychology, vol. 36, no. 3, pp. 321-339, 2005.

[56] F. F. Brunel and M. R. Nelson, "Explaining gendered responses to "help-self" and "help-others" charity ad appeals: the mediating role of world-views," Journal of Advertising, vol. 29, no. 3, pp. 15-28, 2000.

[57] D. G. Perry and R. E. Pauletti, "Gender and adolescent development," Journal of Research on Adolescence, vol. 21, no. 1, pp. 61-74, 2011.

[58] J. E. Williams and D. L. Best, "Cross-cultural views of women and men," in Psychology and Culture, W. J. Lonner and R. S. Malpass, Eds., pp. 191-196, Allyn \& Bacon, Boston, MA, 1994.

[59] J. E. Williams, R. C. Satterwhite, and D. L. Best, "Pancultural gender stereotypes revisited: the five factor model," Sex Roles, vol. 40, no. 7/8, pp. 513-525, 1999.

[60] L. J. Koenig, A. M. Isaacs, and J. A. J. Schwartz, "Sex differences in adolescent depression and loneliness: why are boys lonelier if girls are more depressed?," Journal of Research in Personality, vol. 28, no. 1, pp. 27-43, 1994. 